Discrete Comput Geom 33:699-715 (2005)

DOI: $10.1007 / \mathrm{s} 00454-004-1149-8$

\title{
On the $L_{p}$ Minkowski Problem for Polytopes*
}

\author{
Daniel Hug, ${ }^{1}$ Erwin Lutwak, ${ }^{2}$ Deane Yang, ${ }^{2}$ and Gaoyong Zhang ${ }^{2}$ \\ ${ }^{1}$ Mathematisches Institut, Universität Freiburg, \\ Eckerstrasse 1, D-79104 Freiburg, Germany \\ daniel.hug@math.uni-freiburg.de \\ ${ }^{2}$ Department of Mathematics, Polytechnic University, \\ Six Metrotech Center, Brooklyn, NY 11201, USA \\ \{elutwak,dyang,gzhang\}@poly.edu
}

\begin{abstract}
Two new approaches are presented to establish the existence of polytopal solutions to the discrete-data $L_{p}$ Minkowski problem for all $p>1$.
\end{abstract}

\section{Introduction}

As observed by Schneider [23], the Brunn-Minkowski theory springs from joining the notion of ordinary volume in Euclidean $d$-space, $\mathbb{R}^{d}$, with that of Minkowski combinations of convex bodies. One of the cornerstones of the Brunn-Minkowski theory is the classical Minkowski problem. For polytopes the problem asks for the necessary and sufficient conditions on a set of unit vectors $u_{1}, \ldots, u_{n} \in S^{d-1}$ and a set of real numbers $\alpha_{1}, \ldots, \alpha_{n}>0$ that guarantee the existence of a polytope in $\mathbb{R}^{d}$ with $n$ facets whose outer unit normals are $u_{1}, \ldots, u_{n}$ and such that the facet whose outer unit normal is $u_{i}$ has area (i.e., $(d-1)$-dimensional volume) $\alpha_{i}$. This problem was completely solved by Minkowski himself (see [23] for reference): if the unit vectors do not lie on a closed hemisphere of $S^{d-1}$, then a solution (i.e., polytope) exists if and only if

$$
\sum_{i=0}^{n} \alpha_{i} u_{i}=0 .
$$

In addition, the solution is unique up to a translation.

In the middle of the last century, Firey (see [23] for references) extended the notion of a Minkowski combination of convex bodies and for each real $p>1$ defined what

* This research was supported, in part, by NSF Grant DMS-0104363. 
are now called Firey-Minkowski $L_{p}$ combinations of convex bodies. A decade ago, in [12], Firey-Minkowski $L_{p}$ combinations were combined with volume and the result was an embryonic $L_{p}$ Brunn-Minkowski theory-often called the Brunn-Minkowski-Firey theory. During the past decade various elements of the $L_{p}$ Brunn-Minkowski theory have attracted increased attention (see, e.g., [3]-[5], [8]-[20], [22], and [24]-[29]).

A central problem within the $L_{p}$ Brunn-Minkowski theory is the $L_{p}$ Minkowski problem. A solution to the $L_{p}$ Minkowski problem when the data is even was given in [12]. This solution turned out to be a critical ingredient in the recently established $L_{p}$ affine Sobolev inequality [18].

Suppose the real index $p$ is fixed. The $L_{p}$ Minkowski problem for polytopes asks for the necessary and sufficient conditions on a set of unit vectors $u_{1}, \ldots, u_{n} \in S^{d-1}$ and a set of real numbers $\alpha_{1}, \ldots, \alpha_{n}>0$ that guarantee the existence of a polytope in $\mathbb{R}^{d}$ containing the origin in its interior with $n$ facets whose outer unit normals are $u_{1}, \ldots, u_{n} \in S^{d-1}$ and such that if the facet with outer unit normal $u_{i}$ has area $a_{i}$ and distance from the origin $h_{i}$, then for all $i$,

$$
h_{i}^{1-p} a_{i}=\alpha_{i} .
$$

Obviously, the case $p=1$ is the classical problem. For $p>1$ uniqueness was established in [12]. The $L_{p}$ Minkowski problem for polytopes is the discrete-data case of the general $L_{p}$ Minkowski problem (described below).

In the discrete even-data case of the problem, outer unit normals $u_{1}, u_{-1}, \ldots, u_{m}, u_{-m}$ are given in antipodal pairs, where $u_{-i}=-u_{i}$, and $\alpha_{-i}=\alpha_{i}$. With the exception of the case $p=d$, existence (and uniqueness) for the even problem was established in [12] for all cases (where the unit vectors do not lie in a closed hemisphere of $S^{d-1}$ ). A normalized version (discussed below) of the problem was proposed and completely solved for $p>1$ and even data in [19]. For $d=2$, the important case $p=0$ of the discrete-data $L_{p}$ Minkowski problem was dealt with by Stancu [26], [27].

A solution to the $L_{p}$ Minkowski problem for $p>d$ was given by Guan and Lin [8] and independently by Chou and Wang [5]. The work of Chou and Wang [5] goes further and solves the problem for polytopes for all $p>1$.

The works of Guan and Lin [8] and Chou and Wang [5] focus on existence and regularity for the $L_{p}$ Minkowski problem. Both works make use of the machinery of the theory of PDEs. The classical Minkowski problem has proven to be of interest to those working in both discrete and computational geometry. It is likely that the $L_{p}$ extension of the problem will in time prove to be of interest to those working in these fields as well. An approach accessible to researchers in convex, discrete, and computational geometry appears to be desirable. This article presents two such approaches.

We begin by recalling the formulation of the $L_{p}$ Minkowski problem in full generality. For a convex body $K$ let $h_{K}=h(K, \cdot): \mathbb{R}^{d} \rightarrow \mathbb{R}$ denote the support function of $K$; i.e., for $x \in \mathbb{R}^{d}$, let $h_{K}(x)=\max _{y \in K}\langle x, y\rangle$, where $\langle x, y\rangle$ is the standard inner product of $x$ and $y$ in $\mathbb{R}^{d}$. The induced norm is denoted by $|\cdot|$. We write $V(K)$ for the $d$-dimensional volume of the convex body $K$ in $\mathbb{R}^{d}$.

The surface area measure, $S(K, \cdot)$, of the convex body $K$ is a Borel measure on the unit sphere, $S^{d-1}:=\left\{x \in \mathbb{R}^{d}:|x|=1\right\}$, such that

$$
\lim _{\varepsilon \rightarrow 0^{+}} \frac{V(K+\varepsilon Q)-V(K)}{\varepsilon}=\int_{S^{d-1}} h_{Q}(u) S(K, d u),
$$


for each convex body $Q$. Here $K+\varepsilon Q$ is the Minkowski combination defined by

$$
h(K+\varepsilon Q, \cdot)=h(K, \cdot)+\varepsilon h(Q, \cdot) .
$$

Existence of the surface area measure was shown by Aleksandrov and Fenchel and Jessen (see [23]). The limit on the left-hand side of (1) is also equal to the special mixed volume $d V_{1}(K, Q):=d V(K, \ldots, K, Q)$, with $d-1$ copies of $K$, and hence

$$
V_{1}(K, Q)=\frac{1}{d} \int_{S^{d-1}} h_{Q}(u) S(K, d u)
$$

see a special case of Theorem 5.1.6 in [23].

The classical Minkowski problem asks for necessary and sufficient conditions for a Borel measure $\mu$ on $S^{d-1}$ (called the data) to be the surface area measure of a convex body $K$. The solution as obtained by Aleksandrov and independently by Fenchel and Jessen (see [23]) is: Corresponding to each Borel measure $\mu$ on $S^{d-1}$ that is not concentrated on a closed hemisphere of $S^{d-1}$, there is a convex body $K$ such that

$$
S(K, \cdot)=\mu
$$

if and only if

$$
\int_{S^{d-1}} u \mu(d u)=0 .
$$

The uniqueness of $K$ (up to translation) is a direct consequence of the Minkowski mixedvolume inequality (see (6.2.2) of [23]) which states that for convex bodies $K, Q$,

$$
V_{1}(K, Q) \geq V(K)^{(d-1) / d} V(Q)^{1 / d},
$$

with equality if and only if $K$ is a dilate of $Q$ (after a suitable translation).

Suppose $p>1$ is fixed and $K$ is a convex body that contains the origin in its interior. The $L_{p}$ surface area measure, $S_{p}(K, \cdot)$, of $K$ is a Borel measure on $S^{d-1}$ such that

$$
\lim _{\varepsilon \rightarrow 0^{+}} \frac{V\left(K+_{p} \varepsilon \cdot Q\right)-V(K)}{\varepsilon}=\frac{1}{p} \int_{S^{d-1}} h_{Q}^{p}(u) S_{p}(K, d u),
$$

for each convex body $Q$ that contains the origin in its interior. Here $K+_{p} \varepsilon \cdot Q$ is the Minkowski-Firey $L_{p}$ combination defined by

$$
h\left(K+_{p} \varepsilon \cdot Q, \cdot\right)^{p}=h(K, \cdot)^{p}+\varepsilon h(Q, \cdot)^{p} .
$$

Existence of the $L_{p}$ surface area measure was established in [12] where it was also shown that

$$
S_{p}(K, \cdot)=h_{K}^{1-p} S(K, \cdot) .
$$

It is easily seen that the surface area measure of a convex body (and hence also all the $L_{p}$ surface area measures) cannot be concentrated on a closed hemisphere of $S^{d-1}$.

It turns out that if $P$ is a polytope with outer unit facet normals $u_{1}, \ldots, u_{n}$, then $\left\{u_{1}, \ldots, u_{n}\right\}$ is the support of the measure $S(P, \cdot)$ and $S\left(P,\left\{u_{i}\right\}\right)=a_{i}$, where as before 
$a_{i}$ denotes the area of the facet of $P$ whose outer unit normal is $u_{i}$. Thus, if $P$ contains the origin in its interior, then

$$
S_{p}\left(P,\left\{u_{i}\right\}\right)=h_{i}^{1-p} a_{i},
$$

where as before $h_{i}=h\left(P, u_{i}\right)$.

The $L_{p}$ Minkowski problem asks for necessary and sufficient conditions for a Borel measure $\mu$ on $S^{d-1}$ (the data for the problem) to be the $L_{p}$ surface area measure of a convex body $K$; i.e., given a Borel measure $\mu$ on $S^{d-1}$ that is not concentrated on a closed hemisphere of $S^{d-1}$, what are the necessary and sufficient conditions for the existence of a convex body $K$ that contains the origin in its interior such that

$$
S_{p}(K, \cdot)=\mu
$$

or equivalently

$$
h_{K}^{1-p} S(K, \cdot)=\mu ?
$$

The problem is of interest for all real $p$.

For $p>1$, but $p \neq d$, the uniqueness of $K$ is a direct consequence of the $L_{p}$ Minkowski mixed-volume inequality (established in [12]) which states that if $p>1$, then for convex bodies $K, Q$ that contain the origin in their interior,

$$
V_{p}(K, Q) \geq V(K)^{(d-p) / d} V(Q)^{p / d},
$$

with equality if and only if $K$ is a dilate of $Q$, where

$$
V_{p}(K, Q):=\frac{p}{d} \lim _{\varepsilon \rightarrow 0^{+}} \frac{V\left(K+_{p} \varepsilon \cdot Q\right)-V(K)}{\varepsilon} .
$$

The existence of the limit is proved in [12].

In [12] it was shown that if $\mu$ is an even Borel measure (i.e., assumes the same values on antipodal Borel sets) that is not concentrated on a closed hemisphere of $S^{d-1}$, then for each $p>1$, there exists a unique convex body $K_{p}$ that is symmetric about the origin such that

$$
S_{p}\left(K_{p}, \cdot\right)=\mu,
$$

provided $p \neq d$. The $L_{p}$ Minkowski problem as originally formulated cannot be solved for all even measures when $p=d$. The following normalized version of the $L_{p}$ Minkowski problem was formulated in [19]: What are the necessary and sufficient conditions on a Borel measure $\mu$ to guarantee the existence of a convex body $K_{p}^{*}$ containing the origin in its interior, such that

$$
\frac{1}{V\left(K_{p}^{*}\right)} S_{p}\left(K_{p}^{*}, \cdot\right)=\mu ?
$$

For all real $p \neq d$ the two versions of the problems are equivalent in that

$$
K_{p}=V\left(K_{p}^{*}\right)^{1 /(p-d)} K_{p}^{*}
$$


or equivalently

$$
K_{p}^{*}=V\left(K_{p}\right)^{-1 / p} K_{p}
$$

It was shown in [19] that the normalized $L_{p}$ Minkowski problem has a solution for all $p>1$ if the data measure is even (again assuming the measure is not concentrated on a subsphere of $S^{d-1}$ ).

It is the aim of this note to present two alternate approaches to the Minkowski problem which show that when the data is a discrete measure, the normalized version of the $L_{p}$ Minkowski problem always has a solution (assuming, as usual, that the measure is not concentrated on a closed hemisphere of $S^{d-1}$ ). It is important to emphasize that all of our results for $p>d$ were first obtained by Guan and Lin [8] and independently by Chou and Wang [5], and all of our results for $p>1$ were first obtained by Chou and Wang [5]. The sole aim of our work is to present polytopal approaches easily accessible to the convex, discrete, and computational geometry community.

Since the classical case $p=1$ has been completely solved, we restrict our attention to $p>1$. Thus, throughout we always assume that the index $p>1$.

\section{Main Results}

Let $\mathcal{K}^{d}$ denote the space of compact convex subsets of $\mathbb{R}^{d}$ with nonempty interiors, and let $\mathcal{P}^{d}$ denote the subset of convex polytopes. The members of $\mathcal{K}^{d}$ are called convex bodies. We write $\mathcal{K}_{0}^{d}$ for the set of convex bodies which contain the origin as an interior point, and put $\mathcal{P}_{0}^{d}:=\mathcal{P}^{d} \cap \mathcal{K}_{0}^{d}$.

For $K \in \mathcal{K}^{d}$, let $F(K, u)$ denote the support set of $K$ with exterior unit normal vector $u$, i.e., $F(K, u)=\{x \in K:\langle x, u\rangle=h(K, u)\}$. The $(d-1)$-dimensional support sets of a polytope $P \in \mathcal{P}^{d}$ are called the facets of $P$. If $P \in \mathcal{P}^{d}$ has facets $F\left(P, u_{i}\right)$ with areas $a_{i}, i=1, \ldots, n$, then $S(P, \cdot)$ is the discrete measure

$$
S(P, \cdot)=\sum_{i=1}^{n} a_{i} \delta_{u_{i}}
$$

with (finite) support $\left\{u_{1}, \ldots, u_{n}\right\}$ and $S\left(P,\left\{u_{i}\right\}\right)=a_{i}$, for each $i=1, \ldots, n$, and where $\delta_{u_{i}}$ denotes the probability measure with unit point mass at $u_{i}$.

Just as the $L_{p}$ surface area measure of a convex body $K \in \mathcal{K}_{0}^{d}$ satisfies

$$
S_{p}(K, \cdot)=h(K, \cdot)^{1-p} S(K, \cdot),
$$

the normalized $L_{p}$ surface area measure of $K$ is defined by

$$
S_{p}^{*}(K, \cdot):=\frac{h(K, \cdot)^{1-p}}{V(K)} S(K, \cdot)
$$

A convex body $K$ is uniquely determined by its $L_{p}$ surface area measure if $p>1$ and $p \neq d$ (for $p=d$ one has uniqueness up to a dilation), uniqueness holds for the normalized $L_{p}$ surface area measure and all $p>1$. 
Again for a polytope $P \in \mathcal{P}_{0}^{d}$ with outer unit facet normals $u_{1}, \ldots, u_{n}$ and facet areas $a_{1}, \ldots, a_{n}>0, i=1, \ldots, n$, the discrete measures $S_{p}(P, \cdot)$ and $S_{p}^{*}(P, \cdot)$ are given by

$$
S_{p}(P, \cdot)=\sum_{i=1}^{n} h\left(P, u_{i}\right)^{1-p} a_{i} \delta_{u_{i}}
$$

and

$$
S_{p}^{*}(P, \cdot)=\sum_{i=1}^{n} \frac{h\left(P, u_{i}\right)^{1-p}}{V(P)} a_{i} \delta_{u_{i}} .
$$

In the case of a discrete measure $\mu=\sum_{j=1}^{n} \alpha_{j} \delta_{u_{j}}$ with unit vectors $u_{1}, \ldots, u_{n}$ not contained in a closed hemisphere and $\alpha_{1}, \ldots, \alpha_{n}>0$, any solution of the $L_{p}$ Minkowski problem for the data $\mu$ is necessarily a polytope with facet normals $u_{1}, \ldots, u_{n}$ (see Theorem 4.6.4 of [23]). The main step in our approach to the $L_{p}$ Minkowski problem for general measures and general convex bodies is to solve first the $L_{p}$ Minkowski problem for discrete measures and polytopes.

Theorem 1.1. Suppose $u_{1}, \ldots, u_{n} \in S^{d-1}$ are not contained in a closed hemisphere and $\alpha_{1}, \ldots, \alpha_{n}$ are positive real numbers. Then, for each $p>1$, there exists a unique polytope $P \in \mathcal{P}_{0}^{d}$ such that

$$
\sum_{j=1}^{n} \alpha_{j} \delta_{u_{j}}=\frac{h(P, \cdot)^{1-p}}{V(P)} S(P, \cdot) .
$$

From Theorem 1.1, we deduce the corresponding result for the $L_{p}$ Minkowski problem involving discrete measures and polytopes.

Theorem 1.2. Suppose $u_{1}, \ldots, u_{n} \in S^{d-1}$ are not contained in a closed hemisphere and $\alpha_{1}, \ldots, \alpha_{n}$ are positive real numbers. Then, for each $p>1$, with $p \neq d$, there exists a unique polytope $P \in \mathcal{P}_{0}^{d}$ such that

$$
\sum_{j=1}^{n} \alpha_{j} \delta_{u_{j}}=h(P, \cdot)^{1-p} S(P, \cdot)
$$

The extension of Theorem 1.1 to general measures can be obtained by approximating with discrete measures. For each approximating discrete measure, we get a polytope as the solution of the discrete $L_{p}$ Minkowski problem. We then show that a subsequence of these polytopes must converge. Unfortunately, the limit body may well have the origin on its boundary. For $p \geq d$, we employ an additional argument to see that this does not occur.

Theorem 1.3. Let $\mu$ be a Borel measure on $S^{d-1}$ whose support is not contained in a closed hemisphere of $S^{d-1}$. Then, for $p>1$, there exists a unique convex body $K \in \mathcal{K}^{d}$ with $0 \in K$ such that

$$
V(K) h(K, \cdot)^{p-1} \mu=S(K, \cdot)
$$

moreover, $K \in \mathcal{K}_{0}^{d}$ if $p \geq d$. 
In Section 4 we show that for each $p \in(1, d)$ there is a Borel measure $\mu_{p}$ on $S^{d-1}$ whose support is not contained in a closed hemisphere of $S^{d-1}$ for which the convex body $K_{p} \in \mathcal{K}^{d}$ with the property that

$$
V\left(K_{p}\right) h\left(K_{p}, \cdot\right)^{p-1} \mu_{p}=S\left(K_{p}, \cdot\right)
$$

is such that 0 is a boundary point of $K_{p}$.

The equivalence of the $L_{p}$ Minkowski problem and its normalized version lets us deduce from Theorem 1.3 the following:

Theorem 1.4. Let $\mu$ be a Borel measure on $S^{d-1}$ whose support is not contained in a closed hemisphere of $S^{d-1}$. Then, for $p>1$ with $p \neq d$, there exists a unique convex body $K \in \mathcal{K}^{d}$ with $0 \in K$ such that

$$
h(K, \cdot)^{p-1} \mu=S(K, \cdot)
$$

moreover, $K \in \mathcal{K}_{0}^{d}$ if $p>d$.

Theorem 1.4 solves the $L_{p}$ Minkowski problem for $p>d$. It would be interesting to find necessary and sufficient conditions for $1<p<d$ which guarantee a solution to the $L_{p}$ Minkowski problem.

\section{Volume and Diameter Bounds}

The following three lemmas will be applied in two different ways. On the one hand, we need them for our first treatment of the $L_{p}$ Minkowski problem for discrete measures and polytopes which is based on Aleksandrov's mapping lemma (see [1]). Here the lemmas are applied in the very special situation where all convex bodies are polytopes containing the origin in their interiors and with the same set of outer unit facet normals and where all measures are discrete with common finite support. Except for Lemma 2.1, the proofs of the lemmas in this special case are not simpler than the ones in the general case. Therefore we present them in the general framework. Then again Lemmas 2.1-2.3 will be required for the solution of the $L_{p}$ Minkowski problem in the case of general convex bodies via an approximation argument.

The next lemma provides a uniqueness result which is used to establish the injectivity of an auxiliary map (see Lemma 3.1) in our first proof of Theorem 1.1. It also yields the uniqueness assertions of Theorems 1.1 and 1.3. Moreover, an estimate established in the course of the proof of Lemma 2.1 is employed in the proof of Lemma 2.2.

Lemma 2.1. Suppose $p>1$, and $K, K^{\prime} \in \mathcal{K}^{d}$ are convex bodies with $0 \in K, K^{\prime}$. If $\mu$ is a Borel measure on $S^{d-1}$ such that $V(K) h(K, \cdot)^{p-1} \mu=S(K, \cdot)$ and $V\left(K^{\prime}\right) h\left(K^{\prime}, \cdot\right)^{p-1} \mu=S\left(K^{\prime}, \cdot\right)$, then $K=K^{\prime}$.

Proof. Let $Q \in \mathcal{K}^{d}$ with $0 \in Q$. Define $\Omega:=\left\{u \in S^{d-1}: h(K, u)>0\right\}$ and $\Omega^{c}:=$ 
$S^{d-1} \backslash \Omega$. First note that

$$
\frac{1}{d} \int_{\Omega} h(K, u) S(K, d u)=\frac{1}{d} \int_{S^{d-1}} h(K, u) S(K, d u)=V_{1}(K, K)=V(K),
$$

and hence $h(K, \cdot) /(d V(K)) S(K, \cdot)$ is a probability measure on $\Omega$. Next note that

$$
S\left(K, \Omega^{c}\right)=V(K) \int_{\Omega^{c}} h(K, u)^{p-1} \mu(d u)=0,
$$

and therefore

$$
V_{1}(K, Q)=\frac{1}{d} \int_{\Omega} h(Q, u) S(K, d u) ;
$$

see (2). These two facts, together with Hölder's inequality, and the assumption $p>1$ give

$$
\begin{aligned}
\left(\frac{1}{d} \int_{S^{d-1}} h(Q, u)^{p} \mu(d u)\right)^{1 / p} & \geq\left(\int_{\Omega}\left(\frac{h(Q, u)}{h(K, u)}\right)^{p} \frac{h(K, u) S(K, d u)}{d V(K)}\right)^{1 / p} \\
& \geq \int_{\Omega} \frac{h(Q, u)}{h(K, u)} \frac{h(K, u) S(K, d u)}{d V(K)} \\
& =\frac{V_{1}(K, Q)}{V(K)} .
\end{aligned}
$$

For $Q=K$ or $Q=K^{\prime}$ the left-hand side of (5) is equal to 1. Hence (5) and Minkowski's mixed-volume inequality (3) imply that

$$
1 \geq \frac{V_{1}\left(K, K^{\prime}\right)}{V(K)} \geq\left(\frac{V\left(K^{\prime}\right)}{V(K)}\right)^{1 / d},
$$

and therefore $V(K) \geq V\left(K^{\prime}\right)$. By symmetry, we get $V(K)=V\left(K^{\prime}\right)$, and thus by the equality conditions of the Minkowski mixed-volume inequality $K=K^{\prime}+t$ for some $t \in \mathbb{R}^{d}$. The assumption and the translation invariance of the surface area measure now yield that

$$
\int_{U}\left[h\left(K^{\prime}+t, u\right)^{p-1}-h\left(K^{\prime}, u\right)^{p-1}\right] \mu(d u)=0
$$

for all Borel sets $U \subset S^{d-1}$. In particular, we may choose $U_{t}:=\left\{u \in S^{d-1}:\langle t, u\rangle>0\right\}$. If $t \neq 0$, then $U_{t}$ is an open hemisphere. Since the support of $\mu$ is not contained in $S^{d-1} \backslash U_{t}$, we see that for $t \neq 0$,

$$
\int_{U_{t}}\left[\left(h\left(K^{\prime}, u\right)+\langle t, u\rangle\right)^{p-1}-h\left(K^{\prime}, u\right)^{p-1}\right] \mu(d u)>0 .
$$

This shows that necessarily $t=0$.

In the following two lemmas we provide a priori bounds for the volume and the diameter of solutions of the $L_{p}$ Minkowski problem. The constant $\kappa_{d}$ denotes the volume of the unit ball $B^{d}$. 
Lemma 2.2. Suppose $\mu$ is a Borel measure on $S^{d-1}$, and the body $K \in \mathcal{K}^{d}$ is such that $0 \in K$ and $V(K) h(K, \cdot)^{p-1} \mu=S(K, \cdot)$. Then

$$
V(K) \geq \kappa_{d}\left(\frac{d}{\mu\left(S^{d-1}\right)}\right)^{d / p}
$$

Proof. Apply (5) with $Q=B^{d}$ and use Minkowski's inequality (3) (i.e., the isoperimetric inequality in this case) to get

$$
\left(\frac{1}{d} \mu\left(S^{d-1}\right)\right)^{1 / p} \geq\left(\frac{\kappa_{d}}{V(K)}\right)^{1 / d},
$$

which is equivalent to the assertion of the lemma.

Subsequently, we set $\alpha_{+}:=\max \{0, \alpha\}$ for $\alpha \in \mathbb{R}$. Further, we write $B^{d}(0, r)$ for the Euclidean ball with center 0 and radius $r \geq 0$.

Lemma 2.3. Suppose $\mu$ is a Borel measure on $S^{d-1}$, and the body $K \in \mathcal{K}^{d}$ is such that $0 \in K$ and $V(K) h(K, \cdot)^{p-1} \mu=S(K, \cdot)$. Assume that for some constant $c_{0}>0$,

$$
\int_{S^{d-1}}\langle u, v\rangle_{+}^{p} \mu(d u) \geq \frac{d}{c_{0}^{p}} \quad \text { for all } \quad v \in S^{d-1} .
$$

Then $K \subset B^{d}\left(0, c_{0}\right)$.

Proof. Define $R:=\max \left\{h(K, v): v \in S^{d-1}\right\}$ and choose $v_{0} \in S^{d-1}$ so that $R=$ $h\left(K, v_{0}\right)$. Then $R\left[0, v_{0}\right] \subset K$, and thus $R\left\langle u, v_{0}\right\rangle_{+} \leq h(K, u)$ for $u \in S^{d-1}$. Hence

$$
\begin{aligned}
\frac{R^{p}}{c_{0}^{p}} \leq R^{p} \frac{1}{d} \int_{S^{d-1}}\left\langle u, v_{0}\right\rangle_{+}^{p} \mu(d u) & \leq \frac{1}{d} \int_{S^{d-1}} h(K, u)^{p} \mu(d u) \\
& =\frac{1}{d} \int_{S^{d-1}} h(K, u) h(K, u)^{p-1} \mu(d u) \\
& =\frac{1}{d V(K)} \int_{S^{d-1}} h(K, u) S(K, d u)=1,
\end{aligned}
$$

which gives $R \leq c_{0}$.

\section{The $L_{p}$ Minkowski Problem for Polytopes}

In this section we describe two different approaches to Theorem 1.1. The first proof is based on the following auxiliary result, which is a minor modification of Aleksandrov's mapping lemma. We include the proof for the sake of completeness. Note that Aleksandrov used his mapping lemma to solve the classical Minkowski problem for polytopes. 
Lemma 3.1. Let $A, B \subset \mathbb{R}^{n}$ be nonempty open sets, let $B$ be connected, and let $\varphi: A \rightarrow B$ be an injective, continuous map. Assume that any sequence $\left(x^{i}\right)_{i \in \mathbb{N}}$ in $A$ with $\varphi\left(x^{i}\right) \rightarrow b \in B$ as $i \rightarrow \infty$ has a subsequence convergent in $A$. Then $\varphi$ is surjective.

Proof. Since $\varphi(A) \subset B$ is nonempty, it is sufficient to show that $\varphi(A)$ is open and closed in $B$.

Let $b^{i} \in \varphi(A), i \in \mathbb{N}$, with $b^{i} \rightarrow b \in B$ as $i \rightarrow \infty$ be given. Then there are $x^{i} \in A$ such that $\varphi\left(x^{i}\right)=b^{i}$ for $i \in \mathbb{N}$. By assumption, there is a subsequence $\left(x^{i_{j}}\right)_{j \in \mathbb{N}}$ with $x^{i_{j}} \rightarrow x \in A$ as $j \rightarrow \infty$. Since $\varphi$ is continuous, $\varphi\left(x^{i_{j}}\right) \rightarrow \varphi(x)$ and therefore $b=\varphi(x)$. Hence $\varphi(A)$ is closed in $B$.

Since $A$ is open in $\mathbb{R}^{n}$ and $\varphi$ is continuous and injective, $\varphi(A)$ is open in $B$ by the theorem of the invariance of domain (see Theorem 36.5 of [21] or Theorem 4.3 of [6]).

In the following we write $H_{u, t}^{-}:=\left\{y \in \mathbb{R}^{d}:\langle y, u\rangle \leq t\right\}$ for the halfspace with (exterior) normal vector $u \in S^{d-1}$ and distance $t \geq 0$ from the origin.

For our first proof of Theorem 1.1, we can assume that the given vectors $u_{1}, \ldots, u_{n}$ are pairwise distinct and not contained in a closed hemisphere. Let $\mathbb{R}_{+}^{n}$ be the set of all $x=\left(x_{1}, \ldots, x_{n}\right) \in \mathbb{R}^{n}$ with positive components. For $x \in \mathbb{R}_{+}^{n}$, we define the (compact, convex) polytope

$$
P(x):=\bigcap_{j=1}^{n} H_{u_{j}, x_{j}}^{-}
$$

The compactness of $P(x)$ is implied by the assumption that $u_{1}, \ldots, u_{n}$ are not contained in a closed hemisphere. Since $x \in \mathbb{R}_{+}^{n}, 0$ is an interior point of $P(x)$. Further, we remark that $x \mapsto P(x), x \in \mathbb{R}_{+}^{n}$, is continuous with respect to the Hausdorff metric (see p. 57 of [23]). We put $B:=\mathbb{R}_{+}^{n}$ and define

$$
A:=\left\{x \in \mathbb{R}_{+}^{n}: S\left(P(x),\left\{u_{j}\right\}\right)>0 \text { for } j=1, \ldots, n\right\} .
$$

Note that if $x \in A$, then $x_{j}=h\left(P(x), u_{j}\right)$ for $j=1, \ldots, n$. Clearly, $A, B$ are nonempty open subsets of $\mathbb{R}^{n}$ and $B$ is connected. Next we define the map $\varphi: A \rightarrow B$ by $\varphi(x):=$ $b=\left(b_{1}, \ldots, b_{n}\right)$ with

$$
b_{j}:=\frac{h\left(P(x), u_{j}\right)^{1-p}}{V(P(x))} S\left(P(x),\left\{u_{j}\right\}\right)=S_{p}^{*}\left(P(x),\left\{u_{j}\right\}\right), \quad j=1, \ldots, n .
$$

We will show that $\varphi$ satisfies the assumptions of Lemma 3.1 to conclude that $\varphi$ is surjective. The map $\varphi$ is well-defined and continuous. The continuity of $\varphi$ follows from the continuity of the volume and the support function and from the weak continuity of the surface area measure, since $x \mapsto P(x)$ is continuous as well. Next we check that $\varphi$ is injective. Let $x, y \in A$ be such that $\varphi(x)=\varphi(y)$. Then Lemma 2.1 yields that $P(x)=P(y)$. Hence, by the definition of $A, x_{j}=h\left(P(x), u_{j}\right)=h\left(P(y), u_{j}\right)=y_{j}$ for $j=1, \ldots, n$, and thus $x=y$. 
Now let $x^{i} \in A, i \in \mathbb{N}$, be given. Assume that $b^{i}:=\varphi\left(x^{i}\right) \rightarrow b \in B$ as $i \rightarrow \infty$ and put $\mu_{i}:=S_{p}^{*}\left(P\left(x^{i}\right), \cdot\right)$ for $i \in \mathbb{N}$. Since

$$
\mu_{i}\left(S^{d-1}\right)=\sum_{j=1}^{n} \mu_{i}\left(\left\{u_{j}\right\}\right)=\sum_{j=1}^{n} b_{j}^{i} \rightarrow \sum_{j=1}^{n} b_{j}
$$

as $i \rightarrow \infty$, we obtain $\mu_{i}\left(S^{d-1}\right) \leq c_{1}<\infty$ for all $i \in \mathbb{N}$. Hence, by Lemma 2.2 there is a constant $c_{2}>0$ such that, for $i \in \mathbb{N}$,

$$
V\left(P\left(x^{i}\right)\right) \geq c_{2}>0 .
$$

For the discrete measure $\mu:=\sum_{j=1}^{n} b_{j} \delta_{u_{j}}$ we have $\mu_{i} \rightarrow \mu$ weakly as $i \rightarrow \infty$. The functions $f_{i}, f$ defined by

$$
f_{i}(v):=\int_{S^{d-1}}\langle u, v\rangle_{+}^{p} \mu_{i}(d v), \quad f(v):=\int_{S^{d-1}}\langle u, v\rangle_{+}^{p} \mu(d v),
$$

$v \in S^{d-1}$, are continuous and positive since the support of $\mu_{i}, \mu$ is not contained in a closed hemisphere. Since $f_{i}$ converges uniformly to $f$ as $i \rightarrow \infty$ and the sphere is compact, there is a constant $c_{3}>0$ such that $f_{i}(v) \geq c_{3}$ for all $v \in S^{d-1}$ and $i \in \mathbb{N}$. Lemma 2.3 now implies that there is a constant $c_{4}$ such that, for $i \in \mathbb{N}$,

$$
P\left(x^{i}\right) \subset B^{d}\left(0, c_{4}\right) .
$$

By (7) there exists a convergent subsequence of $P\left(x^{i}\right), i \in \mathbb{N}$. To simplify the notation, we assume that $P\left(x^{i}\right) \rightarrow P \in \mathcal{P}^{d}$ as $i \rightarrow \infty$. Note that by (6) $P$ has indeed nonempty interior. Clearly, $0 \in P$ and the facets of $P$ are among the support sets $F\left(P, u_{1}\right), \ldots, F\left(P, u_{n}\right)$ of $P$ with normal vectors $u_{1}, \ldots, u_{n}$. We next show that $0 \in \operatorname{int}(P)$. For this, assume that 0 is a boundary point of $P$. Then there is a facet $F\left(P, u_{j}\right)$ of $P$ with $0 \in F\left(P, u_{j}\right)$ and $S\left(P,\left\{u_{j}\right\}\right)>0$, and therefore $h\left(P, u_{j}\right)=0$. Consequently, we get $h\left(P\left(x^{i}\right), u_{j}\right) \rightarrow 0$ and $S\left(P\left(x^{i}\right),\left\{u_{j}\right\}\right) \nrightarrow \rightarrow 0$, as $i \rightarrow \infty$. In view of (7) this implies that

$$
b_{j}^{i}=V\left(P\left(x^{i}\right)\right)^{-1} \frac{S\left(P\left(x^{i}\right),\left\{u_{j}\right\}\right)}{h\left(P\left(x^{i}\right), u_{j}\right)^{p-1}} \rightarrow \infty
$$

as $i \rightarrow \infty$, a contradiction.

Since $0 \in \operatorname{int}(P)$, we conclude that $h\left(P\left(x^{i}\right), u_{j}\right) \not \rightarrow 0$ as $i \rightarrow \infty$, for $j=1, \ldots, n$, and therefore also $S\left(P\left(x^{i}\right),\left\{u_{j}\right\}\right) \not \rightarrow 0$; here we use (6) and $b_{j}^{i} \rightarrow b_{j} \neq 0$ as $i \rightarrow \infty$. This finally shows that $S\left(P,\left\{u_{j}\right\}\right)>0$ for $j=1, \ldots, n$.

Thus we get $P=P(x)$ for $x:=\left(h\left(P, u_{1}\right), \ldots, h\left(P, u_{n}\right)\right) \in A$ and $x^{i} \rightarrow x$ as $i \rightarrow \infty$.

Now Lemma 3.1 shows that $\varphi$ is surjective, which implies the existence assertion of the theorem. Uniqueness has already been established in Lemma 2.1.

We now give a second, variational proof of Theorem 1.1. An obvious advantage of this approach is that it may be turned into a nonlinear reconstruction algorithm for retrieving a convex polytope from its $L_{p}$ surface area measure. The main difficulty consists in 
showing that the solution of an auxiliary optimization problem is a convex polytope which contains the origin in its interior.

The following lemma will be used to verify that a convex polytope which is defined as the solution of an auxiliary optimization problem is indeed the solution of the normalized $L_{p}$ Minkowski problem stated in Theorem 1.1. Lemma 3.2 can be found on p. 280 of [1].

Lemma 3.2. Let $u_{1}, \ldots, u_{n} \in S^{d-1}$ be pairwise distinct vectors which are not contained in a closed hemisphere. For $x \in \mathbb{R}_{+}^{n}$, let $P(x):=\bigcap_{i=1}^{n} H_{u_{i}, x_{i}}^{-}$and $\tilde{V}(x):=$ $V(P(x))$. Then $\tilde{V}$ is of class $C^{1}$ and $\partial_{i} \tilde{V}(x)=S\left(P(x),\left\{u_{i}\right\}\right)$ for $i=1, \ldots, n$.

Proof. The second assertion can be checked by a direct argument. Alternatively, it can be obtained as a very special case of Theorem 6.5.3 in [23]. Here one has to choose $\Omega=\left\{u_{1}, \ldots, u_{n}\right\}$, a positive, continuous function $f: S^{d-1} \rightarrow \mathbb{R}$ with $f\left(u_{j}\right)=x_{j}$, and a continuous function $g_{i}: S^{d-1} \rightarrow \mathbb{R}$ with $g_{i}\left(u_{j}\right)=\delta_{i j}$, for $j=1, \ldots, n$. The first assertion then follows, since $x \mapsto S\left(P(x),\left\{u_{i}\right\}\right)$ is continuous on $\mathbb{R}_{+}^{n}$ (see the first proof of Theorem 1.1).

We start with the second proof of Theorem 1.1. Again we can assume that $u_{1}, \ldots, u_{n}$ are pairwise distinct unit vectors not contained in a closed hemisphere. Let $\alpha_{1}, \ldots, \alpha_{n}>$ 0 be fixed. We denote by $\mathbb{R}_{\star}^{n}$ the set of all $x=\left(x_{1}, \ldots, x_{n}\right) \in \mathbb{R}^{n}$ with nonnegative components. Then we define the compact set

$$
M:=\left\{x \in \mathbb{R}_{\star}^{n}: \phi(x)=1\right\},
$$

where

$$
\phi(x):=\frac{1}{d} \sum_{i=1}^{n} \alpha_{i} x_{i}^{p}
$$

For $x \in M$, we again write $P(x)$ for the convex polytope defined by

$$
P(x):=\bigcap_{i=1}^{n} H_{u_{i}, x_{i}}^{-} .
$$

Clearly, for any $x \in M, 0 \in P(x)$ and $P(x)$ has at most $n$ facets whose outer unit normals are from the set $\left\{u_{1}, \ldots, u_{n}\right\}$. Moreover, $h\left(P(x), u_{i}\right) \leq x_{i}$ with equality if $S\left(P(x),\left\{u_{i}\right\}\right)>0$, for $i=1, \ldots, n$. Since $M$ is compact and the function $x \mapsto$ $V(P(x))=: \tilde{V}(x), x \in M$, is continuous, there is a point $z \in M$ such that $\tilde{V}(x) \leq \tilde{V}(z)$ for all $x \in M$. We will prove that $P(z)$ is the required polytope.

First, we show that

$$
0 \in \operatorname{int}(P(z)) \text {. }
$$

This will be proved by contradiction. Let $h_{i}:=h\left(P(z), u_{i}\right)$ for $i=1, \ldots, n$. Without loss of generality, assume that $h_{1}=\ldots=h_{m}=0$ and $h_{m+1}, \ldots, h_{n}>0$ for some $1 \leq m<n$. Note that $m<n$ is implied by $\tilde{V}(z)>0$. We will show that under this assumption there is some $z_{t} \in M$ such that $\tilde{V}\left(z_{t}\right)>\tilde{V}(z)$, which contradicts the definition of $z$. Pick a small $t>0$ and consider

$$
z_{t}:=\left(\left(z_{1}^{p}+t^{p}\right)^{1 / p}, \ldots,\left(z_{m}^{p}+t^{p}\right)^{1 / p},\left(z_{m+1}^{p}-\alpha t^{p}\right)^{1 / p}, \ldots,\left(z_{n}^{p}-\alpha t^{p}\right)^{1 / p}\right),
$$


where

$$
\alpha:=\frac{\sum_{i=1}^{m} \alpha_{i}}{\sum_{i=m+1}^{n} \alpha_{i}} .
$$

Since $0<h_{i} \leq z_{i}$ for $m+1 \leq i \leq n$, we have $z_{t} \in M$ if $t>0$ is sufficiently small.

Define

$$
P_{t}:=\bigcap_{i=1}^{m} H_{u_{i}, t}^{-} \cap \bigcap_{i=m+1}^{n} H_{u_{i},\left(h_{i}^{p}-\alpha t^{p}\right)^{1 / p}},
$$

hence $P_{0}=P(z), P_{t} \subset P\left(z_{t}\right)$ and $0 \in \operatorname{int}\left(P_{t}\right)$, if $t>0$ is sufficiently small. We put

$$
f_{i}:=S\left(P(z),\left\{u_{i}\right\}\right) \quad \text { and } \quad \Delta_{i}(t):=S\left(P_{t},\left\{u_{i}\right\}\right)-f_{i},
$$

and thus

$$
d V\left(P_{t}\right)=t \sum_{i=1}^{m}\left(f_{i}+\Delta_{i}(t)\right)+\sum_{i=m+1}^{n}\left(h_{i}^{p}-\alpha t^{p}\right)^{1 / p}\left(f_{i}+\Delta_{i}(t)\right)
$$

and

$$
d V_{1}\left(P_{t}, P(z)\right)=0 \sum_{i=1}^{m}\left(f_{i}+\Delta_{i}(t)\right)+\sum_{i=m+1}^{n} h_{i}\left(f_{i}+\Delta_{i}(t)\right),
$$

where (2) is used.

Since an interior point of $P(z)$ is also an interior point of $P_{t}$, if $t>0$ is sufficiently small, it follows that $P_{t} \rightarrow P(z)$ as $t \rightarrow 0^{+}$(see p. 57 of [23]), and therefore $\Delta_{i}(t) \rightarrow 0$ as $t \rightarrow 0^{+}$. From this and since at least one facet is supposed to contain the origin, we deduce that

$$
\begin{aligned}
\lim _{t \rightarrow 0^{+}} \frac{V\left(P_{t}\right)}{}-V_{1}\left(P_{t}, P(z)\right) & \\
= & \frac{1}{d} \lim _{t \rightarrow 0^{+}}\left(\sum_{i=1}^{m} \frac{t-0}{t}\left(f_{i}+\Delta_{i}(t)\right)+\sum_{i=m+1}^{n} \frac{\left(h_{i}^{p}-\alpha t^{p}\right)^{1 / p}-h_{i}}{t}\left(f_{i}+\Delta_{i}(t)\right)\right) \\
= & \frac{1}{d} \sum_{i=1}^{m} f_{i}>0 .
\end{aligned}
$$

Here the assumption $p>1$ enters in a crucial way. By Minkowski's inequality (3) and since $P_{t} \rightarrow P(z)$ as $t \rightarrow 0^{+}$, we get

$$
\begin{aligned}
0<\lim _{t \rightarrow 0^{+}} \frac{V\left(P_{t}\right)-V_{1}\left(P_{t}, P(z)\right)}{t} & \leq \liminf _{t \rightarrow 0^{+}} \frac{V\left(P_{t}\right)-V\left(P_{t}\right)^{1-1 / d} V(P(z))^{1 / d}}{t} \\
& =V(P(z))^{1-1 / d} \liminf _{t \rightarrow 0^{+}} \frac{V\left(P_{t}\right)^{1 / d}-V(P(z))^{1 / d}}{t} .
\end{aligned}
$$

This shows that $V\left(P_{t}\right)>V(P(z))$ if $t>0$ is sufficiently small. Since $P_{t} \subset P\left(z_{t}\right)$, the required contradiction follows.

From (8) it follows that

$$
z \in M_{+}:=\left\{x \in \mathbb{R}_{+}^{n}: \phi(x)=1\right\},
$$


and $\tilde{V}(x) \leq \tilde{V}(z)$ for all $x \in M_{+}$. Hence, by the Lagrange multiplier rule there is some $\lambda \in \mathbb{R}$ such that

$$
\nabla \tilde{V}(z)=\lambda \nabla \phi(z)
$$

The required differentiability of $\tilde{V}$ is ensured by Lemma 3.2, and $\nabla \phi(z) \neq 0$ since $z \in \mathbb{R}_{+}^{n}$ and $\alpha_{1}, \ldots, \alpha_{n}>0 ;$ moreover,

$$
f_{i}=\lambda \frac{1}{d} \alpha_{i} p z_{i}^{p-1}, \quad i=1, \ldots, n,
$$

and thus $\lambda>0$, since $f_{i}>0$ for some $i \in\{1, \ldots, n\}$. We deduce that $f_{i}>0$ and therefore $h\left(P(z), u_{i}\right)=z_{i}$ for all $i=1, \ldots, n$. Since $\phi(z)=1$, we obtain

$$
d V(P(z))=\sum_{i=1}^{n} f_{i} z_{i}=\lambda p \frac{1}{d} \sum_{i=1}^{n} \alpha_{i} z_{i}^{p}=\lambda p .
$$

This shows that, for $i=1, \ldots, n$,

$$
S\left(P(z),\left\{u_{i}\right\}\right)=f_{i}=\frac{d}{p} V(P(z)) \frac{p}{d} \alpha_{i} z_{i}^{p-1}=V(P(z)) h\left(P(z), u_{i}\right)^{p-1} \alpha_{i}>0,
$$

which completes our second proof of Theorem 1.1 .

\section{The General Case}

We now provide a proof of Theorem 1.3. Theorem 1.4 is an immediate consequence by the equivalence between the Minkowski problem and its normalized version, as outlined in the Introduction. Let $\mu$ be a Borel measure on $S^{d-1}$ whose support is not contained in a closed hemisphere. As on pp. 392-393 of [23], one can construct a sequence of discrete measures $\mu_{i}, i \in \mathbb{N}$, such that the support of $\mu_{i}$ is not contained in a closed hemisphere and $\mu_{i} \rightarrow \mu$ weakly as $i \rightarrow \infty$. By Theorem 1.1 , for each $i \in \mathbb{N}$ there exists a polytope $P_{i} \in \mathcal{P}_{0}^{d}$ with

$$
\mu_{i}=\frac{h\left(P_{i}, \cdot\right)^{1-p}}{V\left(P_{i}\right)} S\left(P_{i}, \cdot\right) .
$$

As in the proof of (7), we see that the sequence $P_{i}, i \in \mathbb{N}$, is uniformly bounded. Hence we can assume that $P_{i} \rightarrow K \in \mathcal{K}^{d}$ as $i \rightarrow \infty$ and $0 \in K$. In fact, since $\mu_{i}\left(S^{d-1}\right) \rightarrow \mu\left(S^{d-1}\right)$ as $i \rightarrow \infty$, we get as in the proof of (6) that $V(K)>0$, and thus $K \in \mathcal{K}^{d}$.

For a continuous function $f \in C\left(S^{d-1}\right)$ and $i \in \mathbb{N}$ we have

$$
\int_{S^{d-1}} f(u) V\left(P_{i}\right) h\left(P_{i}, u\right)^{p-1} \mu_{i}(d u)=\int_{S^{d-1}} f(u) S\left(P_{i}, d u\right) .
$$

Since $V\left(P_{i}\right) h\left(P_{i}, \cdot\right)^{p-1} \rightarrow V(K) h(K, \cdot)^{p-1}$ uniformly on $S^{d-1}$ (note that $p-1>0$ ), and since $\mu_{i} \rightarrow \mu$ and $S\left(P_{i}, \cdot\right) \rightarrow S(K, \cdot)$ weakly, as $i \rightarrow \infty$, we obtain from (9) that

$$
\int_{S^{d-1}} f(u) V(K) h(K, u)^{p-1} \mu(d u)=\int_{S^{d-1}} f(u) S(K, d u) .
$$

The existence assertion now follows, since (10) holds for any $f \in C\left(S^{d-1}\right)$.

Uniqueness had been proved in Lemma 2.1 . 
Now we consider the case $p \geq d$. Assume that $K \in \mathcal{K}^{d}$ with $0 \in K$ satisfies $V(K) h(K, \cdot)^{p-1} \mu=S(K, \cdot)$, but $0 \in \partial K$. We derive a contradiction by adapting an argument from [5].

Let $e \in S^{d-1}$ be such that $\partial K$ can locally be represented as the graph of a convex function over (a neighborhood of) $B_{r}:=H_{e, 0} \cap B^{d}(0, r), r>0$, and $K \subset H_{-e, 0}^{-}$(see Theorem 1.12 of [2]), where $H_{e, 0}:=\left\{x \in \mathbb{R}^{d}:\langle x, e\rangle=0\right\}$. Let $\mu_{i}$ and $P_{i} \in \mathcal{P}_{0}^{d}$ be constructed for $\mu$ as in the first part of the proof. In particular, $\mu_{i}\left(S^{d-1}\right) \leq c_{5}<\infty$ and $0 \in \operatorname{int}\left(P_{i}\right)$, for all $i \in \mathbb{N}$, and $P_{i} \rightarrow K$ as $i \rightarrow \infty$ with respect to the Hausdorff metric. Then, for $i \geq i_{0}, \partial P_{i}$ can locally be represented as the graph of a convex function $g_{i}$ over $B_{r}$, and the Lipschitz constants of these functions are uniformly bounded by some constant $L$. We define $G_{i}(y):=y+g_{i}(y) e$ for $y \in B_{r}$, put $\alpha:=p-1$ and write $c_{6}, c_{7}$ for constants independent of $i$ and $r$. Then, for $i \geq i_{0}$,

$$
\begin{aligned}
c_{5} \geq \mu_{i}\left(S^{d-1}\right) & =\frac{1}{V\left(P_{i}\right)} \int_{S^{d-1}} h\left(P_{i}, u\right)^{-\alpha} S\left(P_{i}, d u\right) \\
& \geq c_{6} \int_{G_{i}\left(B_{r}\right)}\left\langle x, \sigma\left(P_{i}, x\right)\right\rangle^{-\alpha} \mathcal{H}^{d-1}(d x),
\end{aligned}
$$

where $\mathcal{H}^{d-1}$ denotes the $(d-1)$-dimensional Hausdorff measure and $\sigma\left(P_{i}, x\right)$ is an exterior unit normal vector of $P_{i}$ at $x \in \partial P_{i}$, which is uniquely determined for $\mathcal{H}^{d-1}$ almost all $x \in \partial P_{i}$. Since $g_{i}$ is Lipschitz on $B_{r}$, the differential $\left(d g_{i}\right)_{y}$ exists for $\mathcal{H}^{d-1}$ almost all $y \in B_{r}$. Let $\left(e_{1}, \ldots, e_{d-1}, e\right)$ be an orthonormal basis of $\mathbb{R}^{d}$. Then we put $\nabla g_{i}(y):=\sum_{j=1}^{d-1}\left(d g_{i}\right)_{y}\left(e_{j}\right) e_{j}$, whenever $\left(d g_{i}\right)_{y}$ exists. Using the area formula and the fact that

$$
\sigma\left(P_{i}, G_{i}(y)\right)=\left(1+\left|\nabla g_{i}(y)\right|^{2}\right)^{-1 / 2}\left(\nabla g_{i}(y)-e\right),
$$

for $\mathcal{H}^{d-1}$-almost all $y \in B_{r}$, we obtain

$$
\begin{aligned}
c_{5} & \geq c_{6} \int_{B_{r}}\left\langle G_{i}(y), \sigma\left(P_{i}, G_{i}(y)\right)\right\rangle^{-\alpha} \sqrt{1+\left|\nabla g_{i}(y)\right|^{2}} \mathcal{H}^{d-1}(d y) \\
& =c_{6} \int_{B_{r}}\left(\left\langle y, \nabla g_{i}(y)\right\rangle-g_{i}(y)\right)^{-\alpha}{\sqrt{1+\left|\nabla g_{i}(y)\right|^{2}}}^{1+\alpha} \mathcal{H}^{d-1}(d y) \\
& \geq c_{6} \int_{B_{r}}\left(\left\langle y, \nabla g_{i}(y)\right\rangle-g_{i}(y)\right)^{-\alpha} \mathcal{H}^{d-1}(d y) .
\end{aligned}
$$

Since

$$
0<\left\langle y, \nabla g_{i}(y)\right\rangle-g_{i}(y) \leq 2 d L|y|+\left|g_{i}(0)\right|,
$$

we further deduce that

$$
c_{5} \geq c_{6} \int_{B_{r}}\left(2 d L|y|+\left|g_{i}(0)\right|\right)^{-\alpha} \mathcal{H}^{d-1}(d y)=c_{7} \int_{0}^{r}\left(2 d L t+\left|g_{i}(0)\right|\right)^{-\alpha} t^{d-2} d t .
$$

Since $\left|g_{i}(0)\right| \rightarrow 0$ as $i \rightarrow \infty$, we can extract a decreasing subsequence of $\left(\left|g_{i}(0)\right|\right)_{i \in \mathbb{N}}$. Hence the monotone convergence theorem yields that

$$
c_{5} \geq c_{7} \int_{0}^{r}(2 d L t)^{-\alpha} t^{d-2} d t,
$$

which implies that $\alpha<d-1$; a contradiction. 
The following example demonstrates that the assumption $p \geq d$ in the second part of the assertion of Theorem 1.3 cannot be omitted.

Example 4.1. We now give an example of a Borel measure $\mu$ on $S^{d-1}$ whose support is not contained in a hemisphere and such that 0 is a boundary point of the uniquely determined convex body $K \in \mathcal{K}^{d}$ for which $V(K) h(K, \cdot)^{p-1} \mu=S(K, \cdot)$. Let $\left(e_{1}, \ldots, e_{d}\right)$ denote an orthonormal basis of $\mathbb{R}^{d}$ such that $\operatorname{span}\left\{e_{1}, \ldots, e_{d-1}\right\}=\mathbb{R}^{d-1} \times\{0\}$.

For $q>1$ we define $g(x):=|x|^{q}$ for $x \in \mathbb{R}^{d-1}$ and

$$
K:=\left\{(x, t) \in \mathbb{R}^{d-1} \times \mathbb{R}: t \geq g(x)\right\} \cap H_{e_{d}, 1}^{-} .
$$

Clearly, $K \in \mathcal{K}^{d}, 0 \in \partial K$, and $\partial K$ is $C^{2}$ in a neighborhood of 0 excluding 0 . The given convex body satisfies $V(K) h(K, \cdot)^{p-1} \mu=S(K, \cdot)$ if

$$
\mu:=\frac{h(K, \cdot)^{1-p}}{V(K)} S(K, \cdot)
$$

defines a finite measure on $S^{d-1}$ and $S\left(K,\left\{-e_{d}\right\}\right)=0$. Since indeed $S\left(K,\left\{-e_{d}\right\}\right)=0$ and $h(K, u)>0$ for $u \in S^{d-1} \backslash\left\{-e_{d}\right\}$, and since $S(K, \cdot)$ is absolutely continuous with respect to the spherical Lebesgue measure (with density function $f_{K}$ ) in a spherical neighborhood of $-e_{d}$, it remains to show that $h(K, \cdot)^{1-p} f_{K}$ is integrable in a spherical neighborhood of $-e_{d}$. For $r \in(0,1)$ we put $B_{r}:=B^{d}(0, r) \cap H_{e_{d}, 0}$. Then we define

$$
a(x):=\left(1+|\nabla g(x)|^{2}\right)^{1 / 2}, \quad x \in B_{r} \backslash\{0\},
$$

where $\nabla g(x):=\sum_{i=1}^{d-1} d g_{x}\left(e_{i}\right) e_{i}=q|x|^{q-2} x$. For $x \in B_{r} \backslash\{0\}$ and

$$
u:=\sigma(K,(x, g(x)))=a(x)^{-1}\left(\nabla g(x)-e_{d}\right),
$$

we get

$$
\begin{gathered}
h(K, u)=\langle(x, g(x)), u\rangle=a(x)^{-1}(q-1)|x|^{q}, \\
f_{K}(u)^{-1}=a(x)^{-(d+1)} \operatorname{det}\left(d^{2} g(x)\right),
\end{gathered}
$$

and hence

$$
h(K, u)^{1-p} f_{K}(u)=(q-1)^{1-p} a(x)^{d+p}|x|^{q(1-p)}\left[\operatorname{det}\left(d^{2} g(x)\right)\right]^{-1} .
$$

A direct computation shows that

$$
\operatorname{det}\left(d^{2} g(x)\right)=q^{d-1}(q-1)|x|^{(q-2)(d-1)},
$$

and therefore

$$
h(K, u)^{1-p} f_{K}(u)=q^{1-d}(q-1)^{-p}|x|^{-[(q-2)(d-1)+q(p-1)]} a(x)^{d+p},
$$

for $x \in B_{r} \backslash\{0\}$ and $u=\sigma(K,(x, g(x)))$. For a given $p \in(1, d)$, we now choose

$$
q:=\frac{2(d-1)}{d+p-2} \in(1,2),
$$

and hence, for $x \in B_{r} \backslash\{0\}$ and $u=\sigma(K,(x, g(x)))$,

$$
h(K, u)^{1-p} f_{K}(u)=q^{1-d}(q-1)^{-p} a(x)^{d+p} .
$$

Since $x \mapsto a(x)$ is bounded on $B_{r} \backslash\{0\}$ and $K$ is strictly convex in a neighborhood of the origin, the required integrability follows. 


\section{References}

1. A.D. Aleksandrov, Konvexe Polyeder, Akademie-Verlag, Berlin (Russian original: 1950), 1958.

2. H. Busemann, Convex Surfaces, Interscience, New York, 1958.

3. S. Campi and P. Gronchi, The $L_{p}$-Busemann-Petty centroid inequality, Adv. Math. 167 (2002), 128-141.

4. S. Campi and P. Gronchi, On the reverse $L p$-Busemann-Petty centroid inequality, Mathematika 49 (2002), 1-11.

5. K.-S. Chou and X.-J. Wang, The $L_{p}$-Minkowski problem and the Minkowski problem in centroaffine geometry, Adv. Math. (in press).

6. K. Deimling, Nonlinear Functional Analysis, Springer-Verlag, Berlin, 1985.

7. R.J. Gardner, Geometric Tomography, Cambridge University Press, Cambridge, 1995.

8. P. Guan and C-S. Lin, On equation $\operatorname{det}\left(u_{i j}+\delta_{i j} u\right)=u^{p} f$ on $S^{n}$, Preprint.

9. C. Hu, X.-N. Ma, and C. Shen, On the Christoffel-Minkowski problem for Firey p-sums, Calc. Var. Partial Differential Equations 21 (2004), 137-155.

10. D. Hug and R. Schneider, Stability results involving surface area measures of convex bodies, Rend. Circ. Mat. Palermo (2) Suppl. 70(II) (2002), 21-51.

11. M. Ludwig, Ellipsoids and matrix valued valuations, Duke Math J. 119 (2003), 159-188.

12. E. Lutwak, The Brunn-Minkowski-Firey theory, I: mixed volumes and the Minkowski problem, J. Differential Geom. 38 (1993), 131-150.

13. E. Lutwak, The Brunn-Minkowski-Firey theory, II: Affine and geominimal surface areas, Adv. Math. 118 (1996), 244-294.

14. E. Lutwak and V. Oliker, On the regularity of solutions to a generalization of the Minkowski problem, J. Differential Geom. 41 (1995), 227-246.

15. E. Lutwak, D. Yang, and G. Zhang, A new ellipsoid associated with convex bodies, Duke Math. J. 104 (2000), 375-390.

16. E. Lutwak, D. Yang, and G. Zhang, $L_{p}$ affine isoperimetric inequalities, J. Differential Geom. 56 (2000), 111-132.

17. E. Lutwak, D. Yang, and G. Zhang, The Cramer-Rao inequality for star bodies, Duke Math. J. 112 (2002), 59-81.

18. E. Lutwak, D. Yang, and G. Zhang, Sharp affine $L_{p}$ Sobolev inequalities, J. Differential Geom. 62 (2002), $17-38$.

19. E. Lutwak, D. Yang, and G. Zhang, On the $L_{p}$-Minkowski problem, Trans. Amer. Math. Soc. 356 (2004), 4359-4370.

20. M. Meyer and E. Werner, On the $p$-affine surface area, Adv. Math. 152 (2000), 288-313.

21. J.R. Munkres, Elements of Algebraic Topology, Addison-Wesley, Menlo Park, California, 1984.

22. D. Ryabogin and A. Zvavitch, The Fourier transform and Firey projections of convex bodies, Indiana Univ. Math. J. 53 (2004), 667-682.

23. R. Schneider, Convex Bodies: the Brunn-Minkowski Theory, Encyclopedia of Mathematics and its Applications 44, Cambridge University Press, Cambridge, 1993.

24. C. Schütt and E. Werner, Polytopes with vertices chosen randomly from the boundary of a convex body, Lecture Notes in Mathematics 1807, Springer-Verlag, New York, 2003, pp. 241-422.

25. C. Schütt and E. Werner, Surface bodies and p-affine surface area, Adv. Math. 187 (2004), 98-145.

26. A. Stancu, The discrete planar $L_{0}$-Minkowski problem, Adv. Math. 167 (2002), 160-174.

27. A. Stancu, On the number of solutions to the discrete two-dimensional $L_{0}$-Minkowski problem, Adv. Math., 180 (2003), 290-323.

28. V. Umanskiy, On solvability of the two dimensional $L_{p}$-Minkowski problem, Adv. Math. 180 (2003), 176-186.

29. E. Werner, The $p$-affine surface area and geometric interpretations, Rend. Circ. Mat. Palermo (2) Suppl. 70(IV) (2002), 367-382.

Received September 15, 2003, and in revised form February 2, 2004. Online publication February 9, 2005. 